\title{
Is immunotherapy a viable option in treating mesothelioma?
}
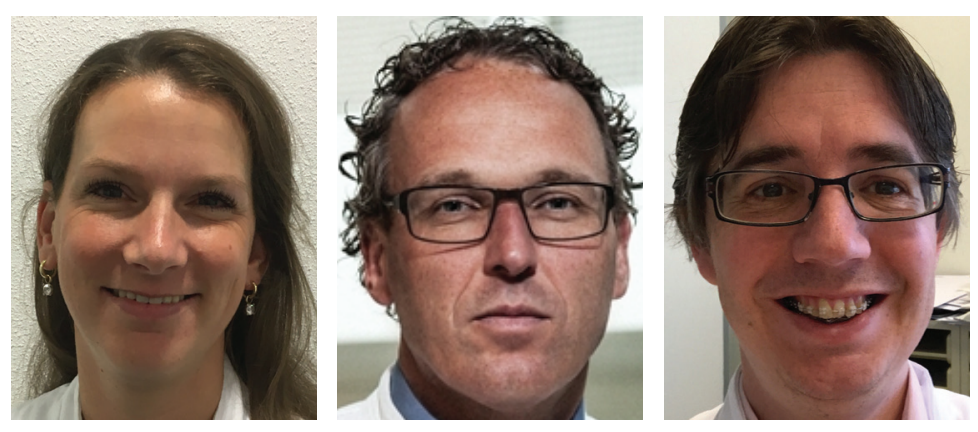

Daphne W Dumoulin', Joachim GJV Aerts' \& Robin Cornelissen*,1

First draft submitted: 17 May 2017; Accepted for publication: 22 May 2017; Published online: 4 August 2017

Over the last years, immunotherapy has been on the rise as an effective treatment option in cancer. For example, immunotherapy targeting $\mathrm{PD}(\mathrm{L})-1$ was found to be effective in non-small-cell lung cancer (NSCLC) [1], with response rates of approximately $20 \%$ in an unselected population and $45 \%$ in a selected population of patients with high PD-L1 expressing tumors [2]. Therefore, anti-PD-(L) 1 has become a standard care for treating NSCLC in second line and in patients with PD-L1 expression $\geq 50 \%$ in first-line therapy.

In malignant pleural mesothelioma, treatment with cisplatinum/pemetrexed resulted in a survival benefit of 3 months [3]. Recently, the addition of bevacizumab resulted in an additional survival benefit of nearly 3 months [4]. Currently, no other therapy has shown activity in a randomized controlled Phase III trial. Therefore, new treatment options are more than desirable. Similar to NSCLC, immunotherapy might have the potency to provide a tumor response with durable survival in mesothelioma [1]. Although, to date no randomized Phase III study using immunotherapy has proven effective in mesothelioma, the research continues extensively. This Editorial aims to answer the question if immunotherapy could be a viable option in mesothelioma by analyzing the immune response needed for an effective immune-mediated tumor killing.

\section{Opportunities to make immunotherapy viable in mesothelioma}

Anticancer immunity is a process consisting of consecutive steps [5]. First, tumor-associated antigens are released by the tumor while it develops. Immature dendritic cells (DCs) can take up these tumor-associated antigens. Afterward, DCs mature, followed by migration to the lymphatic system. There, they can present the captured antigens in presence of major histocompatibility complex-I molecules on the cell membrane to T cells. In turn, these CD8 $T$ cells need to travel to the tumor site, recognize the tumor and invade the tumor and initiate tumor killing.

\section{KEYWORDS}

- CAR T cells $\bullet$ CTLA4 $\bullet$ dendritic cells

- immunotherapy • M2 macrophages

- mesothelioma • PD-1/PD-L1 • tumor microenvironment

"Over the last years, immunotherapy has been on the rise as an effective treatment option in cancer." 
"Of note, the authors of this Editorial have seen a response in a patient participating in this trial, showing the need for more insight into the immune response present in a patient."

\section{- Antigen recognitions \& dendritic cell maturation}

Mesothelioma is a tumor with evident less mutational load than NSCLC, therefore, less tumor antigens are expected, resulting in a lower chance of tumor recognition by DCs [6]. In addition, the tumor microenvironment in mesothelioma has shown to be immune-suppressive with the presence of, for example, M2 macrophages and regulatory $\mathrm{T}$ cells (Tregs), as well as the presence of IL-10, M-CSF, VEGF, prostaglandins and TGF- $\beta$. This also impedes DCs from their potency to act as antigen-presenting cell to T cells [7]. In addition, tumors can inactivate damage-associated molecular pattern, which is needed for DC maturation.

For immunotherapy to be effective, tumor recognition is mandatory. Ex vivo generated activated DCs that have been 'trained' to present tumor antigen could provide a possibility to overcome the problems stated above $[8,9]$.

In 2010, the first clinical trial was conducted with ten mesothelioma patients using autologous tumor lysate-pulsed DCs. After completion of chemotherapy, these mature DCs were injected intradermally and intravenously. Nine patients showed a significant increase in peripheral blood mononuclear cells. Four patients showed an increased cytotoxic activity against autologous tumor cells. This vaccination with DCs was well tolerated [8].

A follow-up trial using cyclophosphamide in addition to DCs resulted in a significant immunoactivity against the tumor [9]. The mechanism of cyclophosphamide will be explained further below. To circumvent the need for autologous tumor material, in the follow-up trial, an allogeneic tumor lysate was used, which resulted in similar antitumor response [10]. Currently, a randomized Phase III study named DENIM with a planned enrollment of 230 patients is about to start.

Another option that is currently tested to activate DCs is vaccination with Listeria [11]. Uptake of the Listeria-based vaccine results in activation of both adapted and innate effector cells, by directly targeting and activating DCs in vivo. Engineered Listeria-based vaccine can express human mesothelin antigen, which is highly expressed in mesothelioma. Uptake of this engineered Listeria by DCs triggers a mesothelin targeted T-cell response.

\section{- Antigen presentation to T cell}

The next step in anticancer immunity is the antigen presenting of DCs to the T cells. In the case that DC maturation is progressing well, optimal T-cell activation may be insufficient due to lower levels of costimulatory ligands and MHC molecules [12]. Activation of T-cell receptor in absence of appropriate costimulation leads to dysfunctional $\mathrm{T}$ cells. By using stimulatory antibodies (such as anti-OX40) [5], anticancer immunity could be increased.

The protein CTLA-4 (cytotoxic T lymphocyte antigen-4) is mainly found on $\mathrm{T}$ cells and has an immune inhibitory effect. T-cell activation decreases when CTLA- 4 is bound to CD-80 and CD-86. Blocking CTLA-4 could therefore be an effective antitumor treatment [13].

CTLA-4 blocking IgG2 antibody tremelimumab has been investigated in mesothelioma in the DETERMINE trial: a Phase IIB placebo-controlled study of tremelimumab in 571 advanced mesothelioma patients as second- and third-line treatment. In terms of patient number, it was the largest immunotherapy study in mesothelioma to date. Unfortunately, no survival benefit was seen in patients using tremelimumab, which might be explained by the treatment in second- and thirdline setting. Of note, the authors of this Editorial have seen a response in a patient participating in this trial, showing the need for more insight into the immune response present in a patient. One treatment probably will not fit all patients [14].

Another option to overcome tumor recognition by $\mathrm{DCs}$ and also T-cell presentation is to produce ex vivo generated $\mathrm{T}$ cells. Chimeric antigen receptors (CARs) T-cell therapy is a form of immunotherapy whereby genetic engineered receptors expressed on T cells bind to a specific tumorassociated antigen and activate T-cell cytotoxicity. These antigen-specific CARs bound $\mathrm{T}$ cells are reinfused to the patient in an effort to eradicate tumors. A murine model showed that administration of CAR T cells intrapleurally needs a 30-fold lower dose to eradicate pleural tumors than intravenously administered CAR T cells, which could be an interesting strategy in mesothelioma patients. The main disadvantage is that CAR T cells only target a specific tumor antigen, allowing for tumor escape mechanism avoiding that single antigen [15]. Another hurdle encountered in the use of CAR $T$ cells in solid tumors is the difficulty in migrating to and adequately penetrating the tumor to unleash their cytotoxic function [16].

\section{- T-cell trafficking \& infiltration}

The next step in anticancer immunity is infiltration of activated $\mathrm{T}$ cells into the tumor. T-cell adhesion molecules are needed for T-cell 
extravasation. These adhesion molecules are downregulated by, for example, VEGF, which is produced in the tumor microenvironment. By targeting VEGF by, for example bevacizumab, several tumor mechanisms are targeted, among which is ineffective T-cell trafficking. The positive result using bevacizumab in the MAPS study might be partially due to more effective T-cell trafficking [4].

\section{- Tumor cell killing}

If the previous stages in the immunity cycle were passed effectively in a patient, it should result in an influx of $\mathrm{CD} 8^{+}$cells in the tumor. This is also called the 'inflamed tumor microenvironment'. However, recognition of MHC complexes on tumor cells by $\mathrm{CD}^{+} \mathrm{T}$ cells is needed for tumor cell killing. T cells are releasing IFN- $\gamma$, which results in releasing of additional cancer antigens but also in expression of multiple coinhibitory receptors such as PD-1, LAG-3, TIM-3 and TIGIT, which together with chronic T-cell receptor signaling induces T-cell dysfunction [17]. An effective tumor treatment could be induced by blocking this negative feedback signal. Several trials have investigated PD-1/PD-L1 inhibitors in mesothelioma. The Keynote 28 is a Phase IB trial in which mesothelioma patients with a PD-L1 $\geq 1 \%$ were treated with pembrolizumab. This trial showed a disease control rate of $72 \%$ and an objective response rate of $20 \%$. The median overall survival was 18 months [18].

The Phase II NivoMes study (nivolumab) and the Phase IB JAVELIN trial (avelumab) showed a disease control rate of approximately $50 \%$ by treating mesothelioma without a PD-L1 expression selection criterion. The overall response rate was $28 \%$ in NivoMes study and $9.4 \%$ in JAVELIN trial $[19,20]$.

Local immune suppression due to immune cells in the tumor microenvironment also impedes effective tumor killing. Although M1 macrophages protect the host by producing IL-12 which stimulates the activation of NK and Th1 cells, M2 macrophages downregulate these functions and produce IL-10, which stimulates Tregs and Th2 cells and so suppresses $\mathrm{CD} 8^{+} \mathrm{T}$-cell response [21,22].
Inhibition of $\mathrm{M} 2$ macrophages in mice resulted in reduced monocytes and neoangiogenesis, but without improving survival. However, combination with DC therapy resulted in improved survival and enhanced $\mathrm{CD} 8^{+} \mathrm{T}$ cells, which induces durable antitumor immunity [23].

In addition, Tregs can promote tumor progression by inhibiting $\mathrm{CD} 8^{+} \mathrm{T}$-cell response [22] Low-dose cyclophosphamide can reduce the number of Tregs. This mechanism was demonstrated in combination with DC immunotherapy. This trial was not powered for efficacy of cyclophosphamide alone, however [8].

\section{Conclusion}

As stated above, mesothelioma tumors have several features that can result in inferior outcomes in trials using the current checkpoint inhibitors that have resulted in positive trials in NSCLC. Most mesothelioma tumors are indeed noninflamed, with low T-cell infiltration. In those patients, treatment must focus on initiating this T-cell response. If the tumor is already inflamed or this has been archived by using a form of immunotherapy and no response is seen then treatment must focus on checkpoint inhibition or the tumor microenvironment. Treatment must be tuned to the missing step $(s)$ in the cancer immunity cycle, and therefore combination treatment can be of added value. By using these tools, immunotherapy may prove to be a viable option for mesothelioma patients.

\section{Financial \& competing interests disclosure}

Stock or other ownership: Amphera; consulting or advisory role: Eli Lilly, Bristol-Myers Squibb, Boehringer Ingelheim, Roche; MSD Oncology, speakers' bureau: AstraZenecal MedImmune; research funding: Eli Lilly (Inst); patents, royalties, other intellectual property: tumor cell lysate for dendritic cell therapy; travel, accommodations, expenses: Boehringer Ingelheim, Verastem R. Cornelissen; speakers fee and advisory boards: Roche, BMS, Novartis, Boehringer Ingelheim, Eli Lilly. The authors have no other relevant affiliations or financial involvement with any organization or entity with a financial interest in or financial conflict with the subject matter or materials discussed in the manuscript apart from those disclosed.

No writing assistance was utilized in the production of this manuscript.

\section{References}

1 Lievense LA, Sterman DH, Cornelissen R, Aerts JG. Checkpoint blockade in lung cancer and mesothelioma. Am. J. Respir. Crit. Care
Med. doi:10.1164/rccm.201608-1755CI (2017) (Epub ahead of print).

2 Reck M, Rodríguez-Abreu D, Robinson AG et al. Pembrolizumab versus chemotherapy for
PD-L1-positive non-small-cell lung cancer. N. Engl. J. Med. 375(19), 1823-1833 (2016).

3 Vogelzang NJ, Rusthoven JJ, Symanowski J et al. Phase III study of pemetrexed in 
combination with cisplatin versus cisplatin alone in patients with malignant pleural mesothelioma. J. Clin. Oncol. Off. J. Am. Soc. Clin. Oncol. 21(14), 2636-2644 (2003).

4 Zalcman G, Mazieres J, Margery J et al. Bevacizumab for newly diagnosed pleural mesothelioma in the mesothelioma avastin cisplatin pemetrexed study (MAPS): a randomised, controlled, open-label, Phase 3 trial. Lancet 387(10026), 1405-1414 (2016).

5 Kim JM, Chen DS. Immune escape to PD-L1/PD-1 blockade: seven steps to success (or failure). Ann. Oncol. Off. J. Eur. Soc. Med. Oncol. 27(8), 1492-1504 (2016).

6 Bueno R, Stawiski EW, Goldstein LD et al. Comprehensive genomic analysis of malignant pleural mesothelioma identifies recurrent mutations, gene fusions and splicing alterations. Nat. Genet. 48(4), 407-416 (2016).

7 Cornelissen R, Lievense LA, Heuvers ME et al. Dendritic cell-based immunotherapy in mesothelioma. Immunotherapy 4(10), 1011-1022 (2012).

8 Cornelissen R, Hegmans JPJJ, Maat APWM et al. Extended tumor control after dendritic cell vaccination with low-dose cyclophosphamide as adjuvant treatment in patients with malignant pleural mesothelioma. Am. J. Respir. Crit. Care Med. 193(9), 1023-1031 (2016).

9 Hegmans JP, Veltman JD, Lambers ME et al. Consolidative dendritic cell-based immunotherapy elicits cytotoxicity against malignant mesothelioma. Am. J. Respir. Crit. Care Med. 181(12), 1383-1390 (2010).

10 Aerts J, Cornelissen R, Leest CVD et al. OA13.06 autologous dendritic cells loaded with allogeneic tumor cell lysate (Pheralys ${ }^{\circledR}$ ) in patients with mesothelioma: final results of a Phase I study. J. Thorac. Oncol. 12(1), S295 (2017).

11 Le DT, Brockstedt DG, Nir-Paz R et al. A live-attenuated Listeria vaccine (ANZ-100) and a live-attenuated Listeria vaccine expressing mesothelin (CRS-207) for advanced cancers: Phase I studies of safety and immune induction. Clin. Cancer Res. Off. J. Am. Assoc. Cancer Res. 18(3), 858-868 (2012).

12 Motz GT, Coukos G. Deciphering and reversing tumor immune suppression. Immunity 39(1), 61-73 (2013).

13 Marcq E, Pauwels P, van Meerbeeck JP, Smits ELJ. Targeting immune checkpoints: new opportunity for mesothelioma treatment? Cancer Treat. Rev. 41(10), 914-924 (2015).

14 Kindler HL, Scherpereel A, Calabrò L et al. Tremelimumab as second- or third-line treatment of unresectable malignant mesothelioma (MM): results from the global, double-blind, placebo-controlled DETERMINE study. J. Clin. Oncol. 34(Suppl. Abstract 8502) doi: 10.1200/ JCO.2016.34.15_suppl.8502 (2016).

15 Mayor M, Zeltsman M, McGee E, Adusumilli PS. A regional approach for CAR T-cell therapy for mesothelioma: from mouse models to clinical trial. Immunotherapy 8(5), 491-494 (2016).

16 Yong CSM, Dardalhon V, Devaud C, Taylor N, Darcy PK, Kershaw MH. CAR T-cell therapy of solid tumors. Immunol. Cell Biol. 95(4), 356-363 (2017).

17 Marcq E, Siozopoulou V, De Waele J et al. Prognostic and predictive aspects of the tumor immune microenvironment and immune checkpoints in malignant pleural mesothelioma. Oncoimmunology 6(1), e1261241 (2017).

18 Alley EW, Lopez J, Santoro A et al. Clinical safety and activity of pembrolizumab in patients with malignant pleural mesothelioma (KEYNOTE-028): preliminary results from a non-randomised, open-label, Phase $1 \mathrm{~b}$ trial. Lancet Oncol. 18 (6), 623-630 (2017).

19 Quispel-Janssen J, Zago G, Schouten R et al. OA13.01 A Phase II study of nivolumab in malignant pleural mesothelioma (NivoMes): with translational research (TR) biopies. J. Thorac. Oncol. 12(1), S292-S293 (2017).

20 Hassan R, Thomas A, Patel MR et al. Avelumab (MSB0010718C; anti-PD-L1) in patients with advanced unresectable mesothelioma from the JAVELIN solid tumor Phase Ib trial: Safety, clinical activity, and PD-L1 expression. J. Clin. Oncol. 34(Suppl. abstr 8503) doi: 10.1200/JCO.2016.34.15_ suppl.8503 (2016).

21 Solinas G, Germano G, Mantovani A, Allavena P. Tumor-associated macrophages (TAM) as major players of the cancer-related inflammation. J. Leukoc. Biol. 86(5), 1065-1073 (2009).

22 Lievense LA, Bezemer K, Aerts JG, Hegmans JP. Tumor-associated macrophages in thoracic malignancies. Lung Cancer Amst. Neth. 80 (3), 256-262 (2013).

23 Dammeijer F, Lievense LA, Aerts JGJV et al. Depletion of tumor-associated macrophages with a CSF-1R kinase inhibitor enhances antitumor immunity and survival induced by DC immunotherapy. Cancer Immunol. Res. 5(7), 535-546 (2017). 